\title{
The Beneficial Effects of an Oriental Herbal Complex Supplement on Women's Hair and Scalp Conditions: A 24-week, Randomized, Double-blind, Placebo-controlled Study
}

\author{
Seung Hun Kim ${ }^{1,2}$, Hee Young Jeon ${ }^{2}$, Su Kyung Kim², Hae Kwang Lee ${ }^{2, *}$, Beom Joon Kim ${ }^{1}$ \\ ${ }^{1}$ Department of Dermatology, Chung-Ang University College of Medicine, Seoul, Korea \\ ${ }^{2}$ Amorepacific Corporation Research and Development Center, Gyeonggi, Korea \\ *Corresponding author: haekwang@amorepacific.com
}

\begin{abstract}
Purpose: Diet is an environmental factor with the potential to significantly influence body changes. Up to now, no study has investigated the effects of the black bean-licorice-biotae folium extract on humans. Therefore, in this study, we examined the effects of this extract on hair and scalp - with regard to total hair number, hair diameter, scalp moisture and sebum. Materials and Method: This study was a randomized, double-blind clinical trial with a 28 week duration. At the -4 week time point of test product intake, participants were enrolled according to criteria and underwent a washout period ( 4 weeks). At weeks 0 , subjects were randomly assigned to one of two treatment regimens: (i) placebo product (4 capsules/day, $700 \mathrm{mg} /$ capsule) or (ii) test product ( 4 capsules/day, 700 $\mathrm{mg} /$ capsule). At weeks 0 and 24, we performed a visual assessment, hair number count and hair diameter, scalp hydration level and sebum excretion level. Results: Expert assessment was positive for the test group. Although the effect on the hair density was not significant, it was positive. Additionally, the hair diameter in the test group significantly increased and significantly decreased in the placebo group. For scalp hydration, the effect was only positive. However, for sebum, the complex appeared to help sebum secretion decrease. There were no safety issues. Conclusion: This study shows that this oriental herbal complex supplement (a Black Bean (Glycine max), Licorice (Glycyrrhiza uralensis Fisch), and Biotae Folium (Thuja orientalis) mixture) is probably effective in improving hair and scalp conditions.
\end{abstract}

Keywords: herbal complex, Black Bean (Glycine max), Licorice (Glycyrrhiza uralensis Fisch), Biotae Folium (Thuja orientalis), hair, scalpe

Cite This Article: Seung Hun Kim, Hee Young Jeon, Su Kyung Kim, Hae Kwang Lee, and Beom Joon Kim, "The Beneficial Effects of an Oriental Herbal Complex Supplement on Women's Hair and Scalp Conditions: A 24-week, Randomized, Double-blind, Placebo-controlled Study." Journal of Food and Nutrition Research, vol. 5, no. 5 (2017): 337-341. doi: 10.12691/jfnr-5-5-8.

\section{Introduction}

The hair growth process can be categorized into three phases: anagen (growing phase, 3-6 years), catagen (regression phase, 2-3 weeks), and telogen (resting phase, $2-4$ months) [1,2]. These cycles usually occur approximately 10 to 20 times in a human's life [2]. Approximately 80 to $90 \%$ of hairs are in the anagen phase, approximately 5\% are in the catagen phase and approximately $10 \%$ are in the telogen phase [2]. These cycles are asynchronous and clinical hair loss is defined as losing approximately 50\% of the original hair [1].

The number of people that have problems with their hair and scalp is increasing steadily. These changes have a variety of causes, such as excessive stress, nutrient deficiency, hormonal changes, and scalp physiology changes. Although two FDA-approved hair loss drugs, finasteride and minoxidil, are available, their uses are limited and transient because of unpredictable efficacy and side effects. As mentioned above, because these problems have a variety of causes, more and better treatment options are needed, and alternatives have been proposed based on these causes [5].

Traditionally, topical and oral medicines such as minoxidil and finasteride have been the major means to solve hair problems. However, over the past few years, several so-called nutraceuticals for skin and hair conditions have appeared in the market place [4]. Recently, a nutraceutical supplement based on a new marine complex formulation has been developed to promote hair growth (Viviscal®, Life2good, Inc., Chicago, IL, USA) $[4,8]$.

We have focused on traditional materials, specifically, beans and oriental herbs. Through literature searches and in vitro and in vivo experiment, we have chosen the following few candidates: black bean (Glycine max), 
licorice (Glycyrrhiza uralensis Fisch), and biotae folium (Thuja orientalis). Black beans are known to be effective in the hair as a source of protein because they contain cysteine, an essential component of hair $[6,7]$. The black bean has lecithin, phytoestrogen, unsaturated fatty acids, anthocyanins and a large amount of protein. These ingredients contribute to physiological actions such as antioxidant effects, anti-obesity effects, estrogenic effects, and immune responses. Additionally, the black bean is known to affect blood circulation and nutrition delivery [2]. Through literature searches and in vitro and in vivo tests, we know that licorice and biotae are effective with respect to $5 \alpha$-reductase inhibition, hair cell growth, blood lipid oxidation prevention and blood lipid reduction. Additionally we know that the three-ingredients combination is more effective than each alone or in a two-component combination. In vivo results showed that this mixture supplement is as effective as a topical treatment $[2,3]$.

Diet is an environmental factor with the potential to significantly influence body changes. Up to now, there is no study investigating the effects of the black beanlicorice-biotae folium extract in humans. Therefore, in this study, we examined the effects of this extract on hair and the scalp, including analyses of total hair number, hair diameter, scalp moisture and sebum.

\section{Material and Methods}

\subsection{Study Design}

This study was a randomized double-blind clinical trial with a 28 week duration. At the -4 week time of test product intake, participants were enrolled according to the criteria and underwent a washout period (4 weeks). At 0 weeks, subjects were randomly assigned to one of two treatment regimens: (i) placebo product (4 capsules/day, $700 \mathrm{mg} /$ capsule) or (ii) test product (4 capsule/day, 700 $\mathrm{mg} / \mathrm{capsule})$. The subsequent experiments and safety checks for adverse events are shown in Table 1.

Table 1. Study flow chart

\begin{tabular}{|l|l|l|l|l|l|}
\hline \multicolumn{2}{|l|}{ Visit Time (weeks) } & -4 & 0 & 12 & 24 \\
\hline \multicolumn{2}{|l|}{ Subject Recruitment and Washout } & $\mathrm{O}$ & & & \\
\hline Visual Evaluation by Dermatologists & $\mathrm{O}$ & $\mathrm{O}$ & & $\mathrm{O}$ \\
\hline \multirow{4}{*}{$\begin{array}{l}\text { Instrumental } \\
\text { Measurement }\end{array}$} & Phototrichogram & & $\mathrm{O}$ & & $\mathrm{O}$ \\
\cline { 2 - 7 } & Moisture Pin & & $\mathrm{O}$ & & $\mathrm{O}$ \\
\cline { 2 - 7 } & Sebumeter & & $\mathrm{O}$ & & $\mathrm{O}$ \\
\hline \multirow{5}{*}{ Safety Check } & Survey & & & $\mathrm{O}$ & $\mathrm{O}$ \\
\cline { 2 - 6 } & $\begin{array}{l}\text { Blood/Urine Test } \\
\text { Weight/Pulse/Blood }\end{array}$ & $\mathrm{O}$ & & & $\mathrm{O}$ \\
\hline & Pressure Measurement & $\mathrm{O}$ & & & $\mathrm{O}$ \\
\hline
\end{tabular}

† Blood Test: Hemoglobin, Red Blood Cell, White Blood Cell, platelet, Hematocrit, MCV, MCH, MCHC, Total Protein, Albumin, Bilirubin, Aspartate Aminotransferase, Alanine Aminotransferase, Glucose, Total Cholesterol, $\gamma$-GTP, Blood Urea Nitrogen

$\dagger$ Urine Test: pH, Nitrite, S.G, Protein, Glucose, Ketone, Bilirubin, Blood Urobilinogen.

\subsection{Sample Size}

Sample size was determined according to the formula ' $n=[2 x(Z \alpha+Z \beta) 2 x \sigma 2] / \Delta 2$. In this study, $Z \alpha=1.96$, $Z \beta=0.84, \sigma=2.4803$, and $\Delta=4.0029$ ( $\sigma$ and $\Delta$ were based on a previous report). Then, we applied the dropout rate, $43 \%$, to calculate the suitable number of subjects.

\subsection{Subjects}

Twenty-eight healthy Korean female volunteers were enrolled in this study. Two persons voluntarily withdrew their consent during the wash out period, so there was a total of twenty-six subjects included. Their ages ranged from 26 to 55 years (mean \pm SD: $47.3 \pm 5.9$ ).

\subsection{Inclusion \& Exclusion Criteria, Others}

Inclusion criteria were as follows: Healthy, Korean females aged 18 to 55 years with androgenic alopecia. Exclusion criteria included subjects with any cutaneous disease, systemic diseases, pregnancy and lactation, or history of any surgical treatment (hair, scalp). We excluded one patient who had a history of using related topical- and systemic-products within the preceding 6 months.

Additionally, subjects had to use only provided products (test product, shampoo and conditioner) and were prohibited from using other products and services to care for their hair and scalp. Subjects also had to maintain their regular diet. The subjects were instructed to return any remaining capsules to the investigators, and subjects who failed to take $\geq 80 \%$ of the capsule-intake were disqualified from further participation.

\subsection{Test Products}

Products were prepared as a hard capsule (700 mg/capsule) containing the ingredients listed in Table 2. Commercially available cellulose, lactose mixture, and magnesium stearate were used. Black bean extract, licorice extract and biotae folium extract were prepared by Amorepacific Co. (Korea).

Table 2. Placebo and Test Product

\begin{tabular}{|l|c|c|}
\hline Ingredient & Placebo $(\%)$ & Test (\%) \\
\hline $\begin{array}{l}\text { Black Bean (Glycin max) } \\
\text { *Licorice(Glycyrrhiza uralensis Fisch) } \\
\text { *Biotae Folium(Thuja orientalis) mixture } \\
(2: 1: 1)\end{array}$ & 0.0 & 71.5 \\
\hline Cellulose & 16.5 & 16.5 \\
\hline Lactose Mixture (Lactose:Dextrin=99:1) & 82.5 & 11.0 \\
\hline Magnesium Stearate & 1.0 & 1.0 \\
\hline Total & & \\
\hline
\end{tabular}

The shampoo and conditioner, which were made by Amorepacific Co. and were available on the market, were provided to subjects.

\subsection{Assessment}

\subsubsection{Visual Assessment}

The hair loss improvement score was quantified by two dermatologists at recruitment, 0 weeks and 24 weeks. In 
this evaluation, the score scale was -3 (worst), $-2,-1,0$ (no change), $+1,+2,+3$ (best).

\subsubsection{Measurement of Hair Number and Diameter}

The measuring site was shaved, marked by a tattoo and then photographed at 0 weeks and 24 weeks. The photographs were analyzed using a phototrichogram device VHX-100 (Keyence, Japan).

\subsubsection{Measurement of Scalp Moisture}

The assessment of scalp hydration was carried out using the Moisture Pin (Cortex Technology, Denmark). It has a pin type probe, which is effective in measuring scalp hydration level. Measurements are arbitrarily expressed as indices of hydration, which increase with increasing hydration.

\subsubsection{Measurement of Scalp Sebum}

The sebum test site included the border of the forehead and hair, and assessment of sebum was conducted using the Sebumeter 810 PC (Courage \& Khazaka, Germany). The unit of measure is $\mu \mathrm{g} / \mathrm{cm}^{2}$, which increases with increasing sebum level.

Assessment of the parameters was performed at 0 weeks and 24 weeks of treatment in a controlled environment with a room temperature of $22 \pm 2{ }^{\circ} \mathrm{C}$ and a relative humidity of $50 \pm 10 \%$ after $30 \mathrm{~min}$ of acclimatization.

\subsection{Research Ethics}

This study was performed at Ellead (Korea) in accordance with the Declaration of Helsinki and was approved by the local Institutional Review Board. The participants signed an informed consent form and underwent the required procedures.

\subsection{Statistical Analysis}

Experimental results are presented as the mean \pm SD (standard deviation). Analysis between groups was performed using the t-test using SPSS software (SPSS Inc., USA) and MS Excel software (Microsoft Co., USA). In all tests, p-values <0.05 were considered statistically significant.

\section{Results}

\subsection{Subjects and Dropout}

Twenty-one subjects completed the entire study treatment protocol: placebo group $(n=11)$, test group $(n=10)$. The number of subjects satisfied the sample size requirement. In both groups, the capsule-intake rate was above 99\%. Two persons voluntarily withdrew their consent during the washout period. Two subjects from the placebo group and three subjects from the test group were excluded owing to lack of cooperation. No adverse reactions were recorded for any of the study participants.

\subsection{Visual Assessment of Hair Loss Improvement}

Among the eleven subjects in the placebo group, there were five subjects who showed no changes in hair loss and the other subjects showed worse hair loss. However, in the test group, most of the subjects remained unchanged and two showed improvement in hair loss (Table 4).

\subsection{Effects on Hair Number and Diameter}

As shown in Table 5, Table 6, the hair number in the placebo group decreased with an improvement rate of $2.9 \%$; in the test group, it increased by $2.6 \%$ after 24 weeks. However there was no statistically significant difference. Hair diameter, in the placebo group significantly decreased by $9.05 \%$; in the test group, it significantly increased by $10.93 \%$ after 24 weeks. At 24 weeks, there were significant differences between the placebo and the test groups with respect to hair diameter $(\mathrm{p}<0.05)$.

\subsection{Effect on Scalp Hydration}

In the test group, scalp hydration slightly increased, but the increase was not statistically significant. The improvement rate was $+9.21 \%$. However, in the case of the placebo, the scalp hydration level significantly decreased after 24 weeks $(-22.45 \%)$ (Table 7).

Table 3. Characteristics of the subjects included in this study

\begin{tabular}{|c|c|c|}
\hline & Placebo $(\mathrm{n}=11)$ & Test $(\mathrm{n}=10)$ \\
\hline Age $(\mathrm{y})$ & $49.0 \pm 4.8$ & $48.1 \pm 3.5$ \\
\hline $\mathrm{N}(40 \mathrm{~s} / 50 \mathrm{~s})$ & $11(6 / 5)$ & $10(7 / 3)$ \\
\hline Intake Rate $(\%)$ & $99.6 \pm 1.1$ & $99.6 \pm 0.5$ \\
\hline
\end{tabular}

Table 4. Visual assessment score by dermatologists

\begin{tabular}{|c|c|c|c|}
\hline \multicolumn{2}{|c|}{ Placebo $(\mathrm{n}=11)$} & \multicolumn{2}{c|}{ Test $(\mathrm{n}=10)$} \\
\hline Subjects No. & Score & Subjects No. & Score \\
\hline 3 & -1 & 1 & 0 \\
\hline 7 & -2 & 2 & 0 \\
\hline 9 & -1 & 4 & 0 \\
\hline 10 & -2 & 6 & 0 \\
\hline 11 & 0 & 8 & 0 \\
\hline 12 & -1 & 16 & 0 \\
\hline 15 & 0 & 19 & 0 \\
\hline 17 & 0 & 22 & 0 \\
\hline 21 & 0 & 23 & +1 \\
\hline 24 & 0 & 26 & \\
\hline 25 & -2 & & \\
\hline
\end{tabular}

Table 5. The change in hair number measured by equipment (number/ $\left.\mathrm{cm}^{2}\right)$

\begin{tabular}{|l|c|c|c|}
\hline \multirow{2}{*}{} & \multicolumn{2}{|c|}{ Hair number } & \\
\cline { 2 - 4 } & Placebo $(\mathrm{n}=11)$ & Test $(\mathrm{n}=10)$ & $p$-value (Placebo vs Test) \\
\hline 0 weeks & $109.00 \pm 35.01$ & $115.20 \pm 29.30$ & $>0.05$ \\
\hline 24 weeks & $105.18 \pm 26.24$ & $118.20 \pm 28.29$ & $>0.05$ \\
\hline Improvement Rate (\%) & -3.50 & 2.60 & \\
\hline$p$-value (0 weeks vs 24 weeks) & $>0.05$ & $>0.05$ & \\
\hline
\end{tabular}


Table 6. The change in hair diameter measured by equipment $(\mu \mathrm{m})$

\begin{tabular}{|l|c|c|c|}
\hline \multirow{2}{*}{} & \multicolumn{2}{|c|}{ Hair Diameter } & \multicolumn{2}{|c|}{ Test (n=10) } & $p$-value (Placebo vs Test) \\
\cline { 2 - 4 } 0 weeks & Placebo $(\mathrm{n}=11)$ & $72.82 \pm 17.03$ & $>0.05$ \\
\hline 24 weeks & $77.13 \pm 15.04$ & $80.78 \pm 14.36$ & $<0.05$ \\
\hline Improvement Rate (\%) & $70.15 \pm 10.99$ & 10.93 & $<0.05$ \\
\hline$p$-value (0 weeks vs 24 weeks) & -9.05 & $<0.05$ & \\
\hline
\end{tabular}

Table 7. The change in scalp hydration level measured by equipment (A.U.)

\begin{tabular}{|l|c|c|c|}
\hline \multirow{2}{*}{} & \multicolumn{2}{|c|}{ Scalp Hydration } & \multicolumn{2}{|c|}{ Test (n=10) } & $p$-value (Placebo vs Test) \\
\cline { 2 - 4 } 0 & Placebo $(\mathrm{n}=11)$ & $135.43 \pm 73.78$ & $>0.05$ \\
\hline 24 weeks & $140.27 \pm 59.56$ & $147.90 \pm 60.82$ & $>0.05$ \\
\hline Improvement Rate (\%) & $108.79 \pm 55.29$ & 9.21 & $>0.05$ \\
\hline$p$-value (0 weeks vs 24 weeks) & -22.45 & $<0.05$ & \\
\hline
\end{tabular}

Table 8. The change in upper forehead sebum level measured by equipment $\left(\mu \mathrm{g} / \mathrm{cm}^{2}\right)$

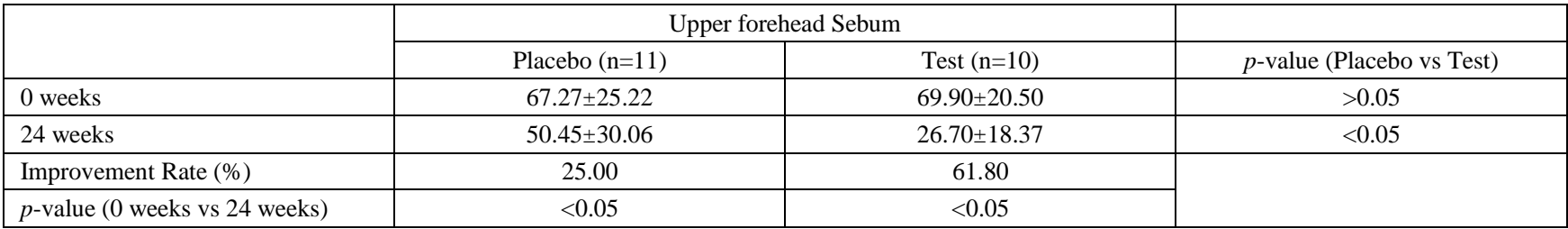

\subsection{Effect on Sebum on Upper Forehead}

In both groups, the sebum level decreased, the decrease was statistically significant $(25.00 \%$ in the placebo group, $61.80 \%$ in the test group. However, the degree of decrease in sebum level in the test group was much greater than the control group. At 24 weeks, there were significant differences between the placebo and the test group with respect to sebum level on the upper forehead $(\mathrm{p}<0.05)$ (Table 8$)$.

\section{Discussion}

To validate the effects in humans, we investigated the efficacy of a Black Bean (Glycine max), Licorice (Glycyrrhiza uralensis Fisch), Biotae Folium (Thuja orientalis) mixture (2:1:1) on hair as well as the scalp in healthy Korean females aged 18-55 years old with androgenic alopecia. We conducted the test over a period of four weeks and a period of 28 weeks. A total of 28 subjects were randomly allocated; seven subjects were excluded from being tested, and a total of 21 subjects (11 subjects in the placebo group, 10 subjects in the test group) completed this study. There were positive results with respect to hair number, hair diameter, scalp hydration, sebum secretion and experts' assessment. Although the effect on hair density was not significant, it was positive. The hair diameter in the test group significantly increased and significantly decreased in the placebo group. For scalp hydration, the results were only positive. However with regard to sebum, the complex appeared to help keep sebum secretion down. There were no safety problems in the study.

In previous in vitro and in vivo studies, we determined that these compounds had effects on dermal papilla cell proliferation, $\mathrm{K}+$ channel modulation (effects on capillary vessels) and hair growth, etc. Additionally, there were increases in total antioxidant capacity and reductions in blood lipid levels. [2,3] Futhermore, sebum level reductions helped to prevent pore-closure due to sebum, and eczema due to fungi which cause inflammation and itching. Perhaps there were improvements in human hair and scalp conditions because of these effects and nutrient supplementation.

Despite several limitations, the present study shows that administration of the combination of black bean (Glycine max), licorice (Glycyrrhiza uralensis Fisch), and biotae folium (Thuja orientalis) is helpful for hair and scalp conditions' improvement. Although the effects may be weaker than medication, long-term food supplementation appears to be safer, and its effects are longer lasting without negative effects.

However, several controlled human studies with large numbers of participants, including a variety of experiment periods and a variety of combinations and dosages of candidate herbs, will be necessary to verify the effects and to better understand their mechanism of action.

\section{Conclusion}

In summary, this study show that this oriental herbal complex supplement is probably effective in improving hair and scalp conditions.

\section{References}

[1] Stenn KS, Combates NJ, Gordon JS, Pardinas JR, Parimoo S, Prouty SM, Hair follicle growth controls. Dermatol Clin 1996, 14, 543-558.

[2] Hee Young Jeon, Seung Hun Kim, Chae Wook Kim, Hyun Jeong Shin, Dae Bang Seo, and Sang Jun Lee, Hair Growth Promoting Effect of Black Soybean Extract In Vitro and In Vivo. Korean J Food Sci Technol 2011, 43: 747-753.

[3] Hee Young Jeon, Seung Hun Kim, Hyun Jeong Shin, Dae Bang Seo, and Sang Jun Lee, Synergistic Effects of the Combination of Oral Herbal Composition and Topical Hair Tonic on Hair Growth in C57BL6 Mice. Korean J Food Sci Technol 2012, 44: 257-262. 
[4] Glynis Ablon, MD, FAAD, A 6-month, randomized, double-blind, placebo-controlled study evaluating the ability of a marine complex supplement to promote hair growth in men with thinning hair. J Cosmet Dermatol 2016, 15: 358-366.

[5] Soojin Park, Weon-Sun Shin, Jinnyoung Ho, Fructus panax ginseng extract promotes hair regeneration in C57BL/6 mice. $J$ Ethnopharmacol 2011, 138: 340-344.

[6] Jones LN, Rivett DE, The role of 18-methylleicosanoic acid in the structure and formation of mammalian hair fibres. Micron 1997, 28: 469-485.

[7] Kovalenko IV, Rippke GR, Hurburgh CR, Determination of amino acid composition of soybeans (Glycine max) by near-infrared spectroscopy. J Agr Food Chem 2006, 24: 3485-3491.

[8] Hornfeldt CS, Holland M, Bucay VW et al, The safety and efficacy of a sustainable marine extract for the treatment of thinning hair: a summary of new clinical research and results form a panel discussion on the problem of thinning hair and current treatments. J Drugs Dermatol 2015, 14: S1-22.

[9] Dayong Wu, Mohsen Meydani, Lynette S Leka, Zachary Nightingale, Garry J Handelman, Jeffrey B Blumberg and Slimin Nikbin Meydani, Effect of dietary supplementation with black currant seed oil on the immune response of healthy elderly subjects. Am J Clin Nutr 1999, 70: 536-543.

[10] D. H. Rushton, Nutrition factors and hair loss. Clin Exp Dermatol 2002, 27: 396-404.

[11] McElwee KJ, Niiyama S, Freyschmidt-Paul P, Wenel E, Kissling S, Sundberg JP, Hoffmann R., Dietary soy oil content and soy-derived phytoestrogen genistein increase resistance to alopecia areata onset in $\mathrm{C} 3 \mathrm{H} / \mathrm{HeJ}$ mice. Exp Dermatol 2003, 12: 30-36.

[12] Takahiro Tsuruki and Masaaki Yoshikawa, Design of Soymetide4 Derivatives to Potentiate the Anti-alopecia Effect. Biosci Biotechnol Biochem 2004, 68: 1139-1141.

[13] Takahiro Tsuruki, Kyoya Takahata, Massaki Yoshikawa, Anti-alopecia mechanisms of soymetide-4, an immunostimulating peptide derived from soy $\beta$-conglycinin. Peptides 2005, 26: 707-711.

[14] Takahiro Tsuruki and Masaaki Yoshikawa, Anti-Alopecia Effect of Gly-Leu-Phe, an Immunostimulating peptide Derived from $\alpha$-Lactalbumin. Biosci Biotechnol Biochem 2005, 69: 1633-1635.

[15] Takahiro Tsuruki, Masaaki Yoshikawa, Orally administered FPRL1 receptor agonist peptide MMK-1 inhibits etoposide-induced alopecia by a mechanism different from intraperitoneally administered MMK-1. Peptides 2006, 27: 820-825.

[16] Mutluay Arslan, Sebahattin Vurucu, Necati Balamtekin, Bulent Unay, Ridvan Akin, Ismail Kurt, Okan Ozcan, The effects of biotin supplementation on serum and liver tissue biotinidase enzyme activity and alopecia in rats which were administrated to valproic acid. Brain Dev 2009, 31: 405-410.

[17] Tongyu Cao Wikramanayake, Elizabeth Alvarez-Connelly, Jessica Simon, Lucia M. Mauro, Javier Guzman, George Elgart, Lawrence A. Schachner, Juan Chen, Lisa R. Plano, Joaquin J. Jimenez, Heat treatment increases the incidence of alopecia areta in the $\mathrm{C} 3 \mathrm{H} / \mathrm{HeJ}$ mouse model. Cell Stress Chaperones 2010, 15: 985-991.

[18] Margarita G. Skalnaya, Vladislav P. Tkachev, Trace elements content and hormonal profiles in women with androgenetic alopecia. J Trace Elem Med Biol 2011, 25S: S50-S53. 